\title{
Survey on Wireless Technology Trade-Offs for the Industrial Internet of Things
}

\author{
Amina Seferagić ${ }^{1, *(\mathbb{D}}$, Jeroen Famaey ${ }^{2}$, Eli De Poorter ${ }^{1}\left(\mathbb{D}\right.$ and Jeroen Hoebeke ${ }^{1}(\mathbb{D}$ \\ 1 IDLab, Department of Information Technology, Ghent University_imec, 9000 Ghent, Belgium; \\ Eli.DePoorter@UGent.be (E.D.P.); Jeroen.Hoebeke@ugent.be (J.H.) \\ 2 IDLab, Department of Computer Science, University of Antwerp-imec, 2000 Antwerp, Belgium; \\ jeroen.famaey@uantwerpen.be \\ * Correspondence: amina.seferagic@ugent.be
}

Received: 7 December 2019; Accepted: 13 January 2020; Published: 15 January 2020

\begin{abstract}
Aside from vast deployment cost reduction, Industrial Wireless Sensor and Actuator Networks (IWSAN) introduce a new level of industrial connectivity. Wireless connection of sensors and actuators in industrial environments not only enables wireless monitoring and actuation, it also enables coordination of production stages, connecting mobile robots and autonomous transport vehicles, as well as localization and tracking of assets. All these opportunities already inspired the development of many wireless technologies in an effort to fully enable Industry 4.0. However, different technologies significantly differ in performance and capabilities, none being capable of supporting all industrial use cases. When designing a network solution, one must be aware of the capabilities and the trade-offs that prospective technologies have. This paper evaluates the technologies potentially suitable for IWSAN solutions covering an entire industrial site with limited infrastructure cost and discusses their trade-offs in an effort to provide information for choosing the most suitable technology for the use case of interest. The comparative discussion presented in this paper aims to enable engineers to choose the most suitable wireless technology for their specific IWSAN deployment.
\end{abstract}

Keywords: Industrial Internet of Things (IIoT); LoRa; IEEE 802.11ah; WiFi HaLow; Time Slotted Channel Hopping (TSCH); Narrowband IoT (NB-IoT); Bluetooth Low Energy (BLE); BLE Long Range; WirelessHART; ISA100.11a

\section{Introduction}

Industrial networks for process automation are deployed in sites which can be hundreds of meters wide, hosting very dense networks consisted of hundreds or thousands of nodes. Harsh industrial environments impose a number of challenges for wireless communications: reliability, fault-tolerance and low latency being the biggest ones. Unpredictable variations in temperature, humidity, vibrations and pressure make the industrial environments harsh, as well as the presence of highly reflective (metal) objects and electromagnetic noise. Even though not much data needs to be communicated in an industrial application, reliability and latency are critical, that is, delivery of all data must be guaranteed in real-time. Wired networks have met these requirements and are being used in spite of the high cost of wiring and the often present installation difficulties (see Figure 1) because wireless solutions are not as robust as their wired counterparts. Industrial automation systems in chemical industry, power plants, oil refineries or underground water supply systems implement complex monitoring and control processes. Thousands of devices send measured values (i.e., temperature, pressure, flow, position) to the actuators that control processes and to the servers that coordinate the production phases. Wiring is generally both challenging and 
costly (cca. $20 \$ / \mathrm{m}$ ): flammable, explosive and hot environments have to be avoided (e.g., in the presence of flammable gases in an oil refinery), remote or unavailable locations are hard to reach and mobile nodes can hardly be connected at all. Although wired networks at this time cannot fully be replaced by wireless networks in this domain, supervision and non-critical control with loose enough requirements could be realized over wireless. In addition, significant constrains that limit the practical deployments of wireless networks in such scenarios are battery capacity and power consumption of the devices. Ideally, communication and power cables can be mitigated to enable a fully wireless solution. For that, the devices should be energy efficient and able to power from a battery for years. Moreover, wireless networks introduce logical benefits that could be used in maintenance and commissioning, such as "plug-n-play" automation architectures to reduce downtime and speed-up tests and "hot-swapping" faulty modules. In addition to control and supervision, global wireless plant coverage could enable localization and tracking of parts in production, coordination of autonomous transport vehicles and mobile robots [1].

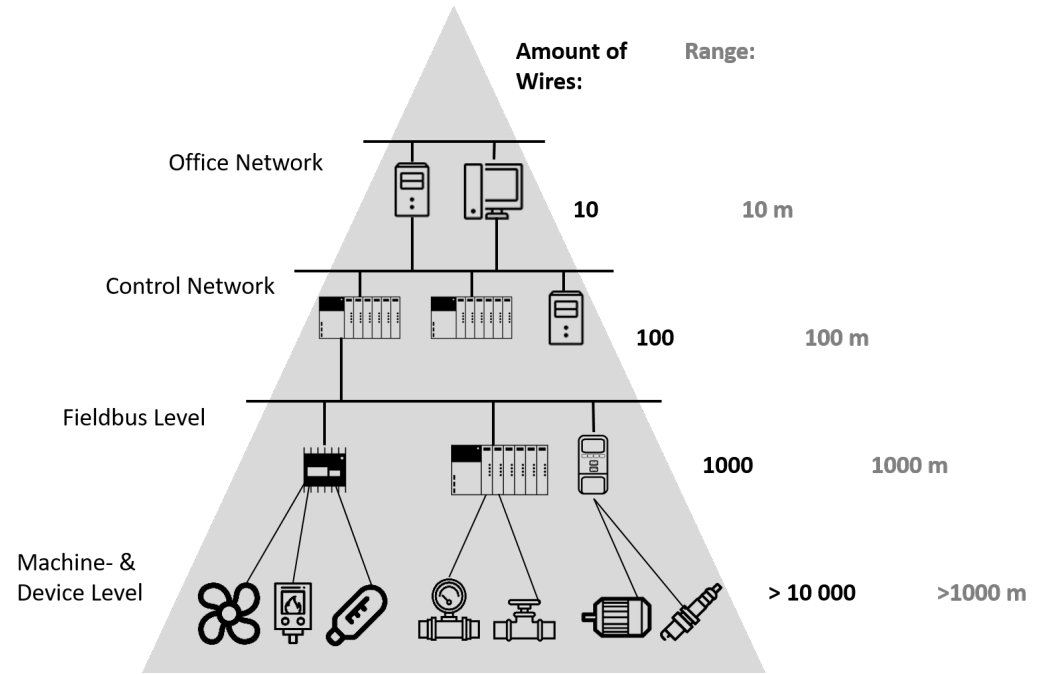

Figure 1. Node density exponentially increases from the top (office network/Internet/Intranet) to the bottom (machine- and device-level) in a typical automation system network hierarchy.

Industrial Wireless Sensor and Actuator Network (IWSAN) are gaining popularity in process industries due to their advantage in lowering infrastructure cost and deployment effort. The advent of Industry 4.0 already resulted in the successful use of IWSANs for monitoring applications and non-critical open-loop control in factory automation. A few new wireless technologies, such as WirelessHP [2], OFDMA wirelesscontrol [3], Real-Time-WiFi [4], Wireless network for Industrial Automation and Process Automation (WIA-PA) [5], can replace extensive wiring on industrial machinery, providing connectivity between machine parts with $\mu$ s order of magnitude latency. Even though they enable reliable and fast communication, the range of such networks is limited to only a few meters, making them unsuitable for broad usage across an entire industrial site in process automation or for reaching remote areas if infrastructure cost has to be kept low. Ranges up to a few hundred meters are feasible with 802.15.4-based technologies such as WirelessHART [6], ISA 100.11a [7], 802.15.4g [8] with Time Slotted Channel Hopping (TSCH) [9] and WIA-PA [10], at the cost of other performance metrics. Sub-GHz wireless technologies, such as LoRa and SigFox, further extend the coverage due to the better signal propagation characteristics (up to $15 \mathrm{~km}$ and $50 \mathrm{~km}$ respectively) but are not suitable for frequent critical traffic given their low data rates (up to $50 \mathrm{kbps}$ and $0.1 \mathrm{kbps}$ respectively) which lead to very long transmission times in both uplink and downlink. In downlink, long transmission times also limit the gateway to serve many nodes, more so considering the duty cycle limitations [11]. Moreover, LoraWAN Class A and Sigfox only allow downlink transmissions that immediately follow uplink, resulting in substantial downlink 
delays due to buffering. NB-IoT experiences downlink delays due to buffering as well, when using the Power Saving Mode (PSM). The existing trade-off between the range and latency varies across different technologies (cf. Figure 2), aiming to cover a variety of use cases. This paper explores the aforementioned trade-offs and the conditions that enable the use of particular Internet of Things (IoT) wireless technologies in heterogeneous sensor-actuator networks for mid-range communication able to cover an industrial site ranging up to more than one kilometer in diameter.

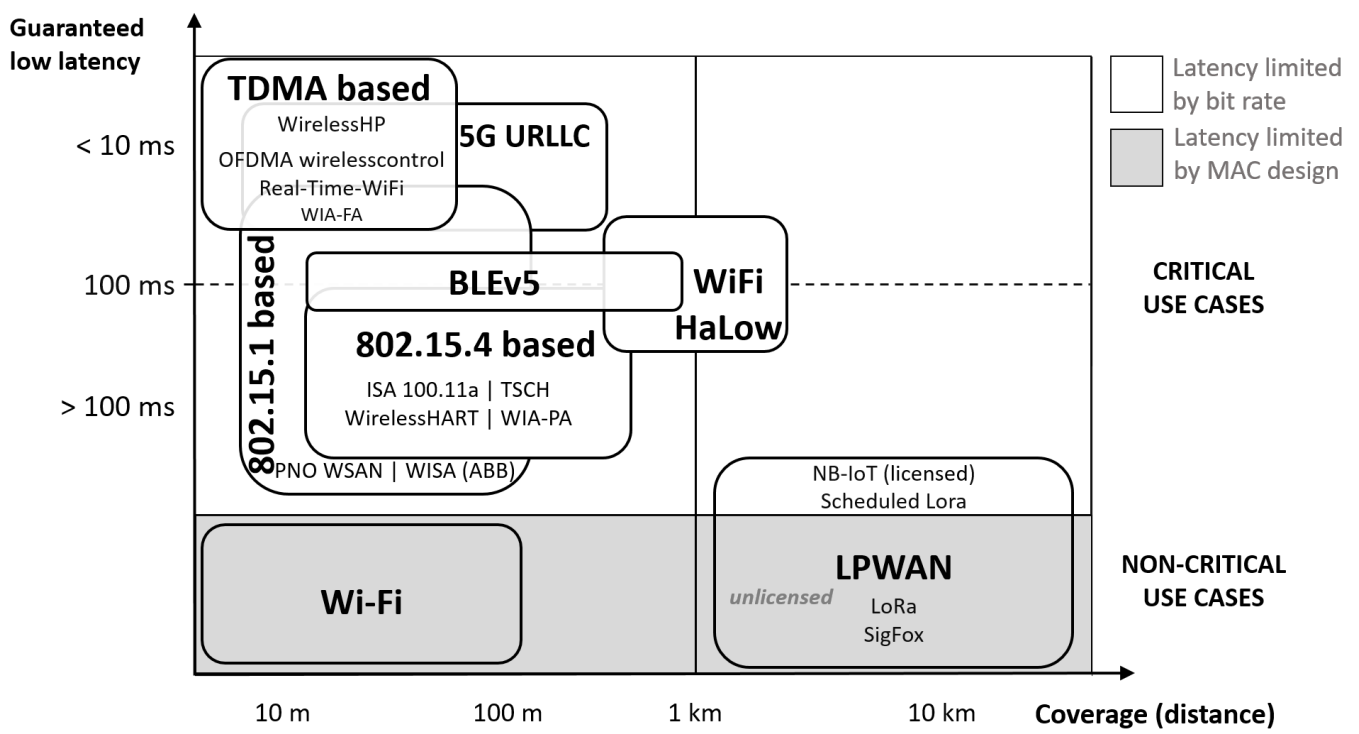

Figure 2. Different wireless technologies have different range/latency capabilities. This article discusses the trade-offs in mid-range technologies that could provide coverage of an entire industrial site (black boxes) with sufficiently low latency.

Wireless Sensor Networks (WSNs) have been evaluated from different perspectives in the state of the art literature. However, significantly smaller amount of research is conducted in the context of IWSANs that have much more strict application requirements. An overview of key issues and challenges of wireless technologies in industrial networks is surveyed in References [1,12-19]. Communication requirements and a general profile of a wireless fieldbus for low level short-range factory automation systems are discussed in Reference [20]. References [13,21] discuss security and Quality of Service (QoS) perspectives of IWSAN in industrial automation. Furthermore, an in-depth review of recent advances in real-time IWSANs for industrial control systems is given in Reference [22], with a focus on WirelessHART. Reference [22] reviews real-time scheduling and analytic techniques for achieving real-time performance in Reference IWSANs. An extensive survey on wireless network design for control systems is presented in Reference [17], briefly reviewing a few of the existing wireless technologies in that context but mostly focusing on the joint design considerations of both control systems and wireless networks. A comparative examination of ZigBee, WirelessHART, ISA100.11a and WIA-PA in terms of network architecture and protocol design in the context of IWSAN is given in Reference [19]. State of the art in Low-Power Wide-Area Network (LPWAN) solutions for Industrial Internet of Things (IIoT) services is explored in Reference [23].

This paper takes a different approach and interprets the wireless standards from the practical standpoint, offering the readers concrete values on achievable sampling rates, energy consumption, scalability and coverage in practice. Both standard specifications and product datasheets provide extensive low level data, and are insufficient on their own without additional empirical research. This paper quantifies the existing trade-offs in wireless technologies for wireless sensor and actuator networks with coverage of at least a couple of hundred meters, able to cover a production site or at least a large part of it. Range is crucial in process automation for all slave nodes to be able to reach a master node, considering that control is typically done by one or few master nodes (controllers) and a 
large number of slave nodes (sensors and actuators) that take part in bidirectional communication with the controller and are spatially distributed over the entire site. This paper presents cost, scalability, latency, reliability, range and energy consumption evaluation of wireless technologies with promising range and latency potential, including LoRa, IEEE 802.11ah (Wi-Fi HaLow), Narrowband-IoT (NB-IoT), WirelessHART, ISA100.11a, Bluetooth Low Energy (BLE) and 802.15.4g physical layer with 802.15.4e TSCH on data-link layer. These technologies offer the possibility of a dense heterogeneous wireless network deployment able to serve both actuators and sensors in critical applications, as well as provide an infrastructure for supervisory traffic. Along with the practical limitations of each technology with respect to the existing trade-offs between latency, throughput, coverage and scalability, a direct projection of the aforementioned wireless technologies to their key performance indicators is made, aiming to enable adequate network design in particular industrial applications.

The remainder of this article is organized as follows. The requirements and challenges that industrial networks must comply with are summarized in Section 2. Section 3 presents a general discussion of the key trade-offs in wireless network design in the context of the requirements, while in Section 4 the discussed trade-offs are quantified for each particular technology. Overall discussion and the experimental evaluation of energy consumption is presented in Section 5. Finally, Section 6 presents the conclusions.

\section{Requirements and Challenges}

The International Society of Automation (ISA) classified industrial systems into six classes [13] on the basis of data urgency and operational requirements. These classes range from critical control systems to monitoring systems, from the strictest requirements to the most relaxed ones respectively:

1. Safety systems-require immediate actions on events (usually in the order of tens or hundreds of $\mu$ s or a few ms).

2. Closed loop regulatory systems - control the system via feedback loops operating either periodically or based on events. They may or may not have stricter timing requirements than safety systems.

3. Closed loop supervisory systems-similar to regulatory systems with the difference that the feedbacks are usually non-critical and event-based, for example, collecting statistical data and reacting only when a certain trend is observed by issuing a notification or alarm.

4. Open loop control systems-where sensors collect data and store it to the central database. An operator (human) analyzes the data and acts upon it if needed.

5. Alerting systems - send periodical or event-based alerts indicating different stages, for example, heating up the boiler and alerting every once in a while to indicate the progress.

6. Information gathering systems - collect the data (logging) and forward the logs to a server. These systems have no immediate operational consequence.

Wireless coverage of the entire industrial site may benefit classes 2-6, whereas class 1 requires a solution combining both ultra-high reliability, redundancy and ultra-low latency, which is infeasible with long range wireless considering the trade-offs. Performance requirements of different classes are depicted in Figure 3. For site-wide coverage, a range of at least a few hundred meters is needed. Site-wide coverage would enable multicasting measurements to several destinations, for example, actuators, supervision systems, databases, enabling the support of different services for several classes of industrial systems, making the network heterogeneous. Thus, site-wide IWSANs need to be scalable enough to accommodate new nodes and provide QoS, considering that they are expected to run for several decades. Different applications require different performance and services, as examples in Table 1 show. 
Table 1. Cycle time and communication range requirements broadly vary over industrial automation use-cases [12,24-26].

\begin{tabular}{lcc}
\hline Application & Range $[\mathrm{m}]$ & Cycle Time \\
\hline Building automation & $10-200$ & $100 \mathrm{~ms}-$ seconds \\
Monitoring and supervision & $100-1000$ & seconds-days \\
Process control & $50-500$ & $10-1000 \mathrm{~ms}$ \\
Factory automation & $10-50$ & $0.5-100 \mathrm{~ms}$ \\
Automotive & $1-10$ & $1-100 \mathrm{~ms}$ \\
Interlocking and control & $50-100$ & $10-250 \mathrm{~ms}$ \\
Power-system protection & $100-10 \mathrm{k}$ & $0.01 \mu \mathrm{s}-50 \mathrm{~ms}$ \\
Event-based control & $10-100$ & $1-100 \mathrm{~ms}$ \\
\hline
\end{tabular}

Besides the key performance requirements illustrated in Figure 3, deployment cost, energy consumption, interoperability, QoS and service differentiation come to focus especially when considering heterogeneous networks. A wired fieldbus network is very expensive to deploy because of tens of kilometers of cables needed to connect devices to their master nodes, the time needed for deployment and the maintenance of such deployment. Lower deployment and implementation costs are the prime motivations for the transition from wired to wireless solutions wherever possible. Among wireless solutions, subscription fees for operator based networks also vary. Operator based solutions are generally not ideal for industrial purposes as the dependence on the operator in case of failure increases the repair time. However, reliable full-duplex operation of wired industrial networks is a large advantage over wireless technologies that are the subject of this article. Namely, reliability inherently suffers in the full-duplex wireless solutions because of the self-interference and increased interference from the neighbours [27]. Opting for half-duplex instead causes the inability to send and receive at the same time on the same channel, which in turn largely increases the latency in wireless networks. IWSANs must operate in real time to serve class two systems. Specifically, closed loop regulatory systems require IWSANs to sample, process and exchange the data between a sensor and an actuator in a time frame that is less than the cycle time of the loop, with typical values ranging from microseconds to hundreds of milliseconds (depending on the concrete process being controlled). Critical applications (classes 1 and 2) also require redundancy, resistance to noise and robustness against failure as they must ensure timely and successful delivery. In addition, the failure of one or a few nodes must not compromise the operation of the network as whole.

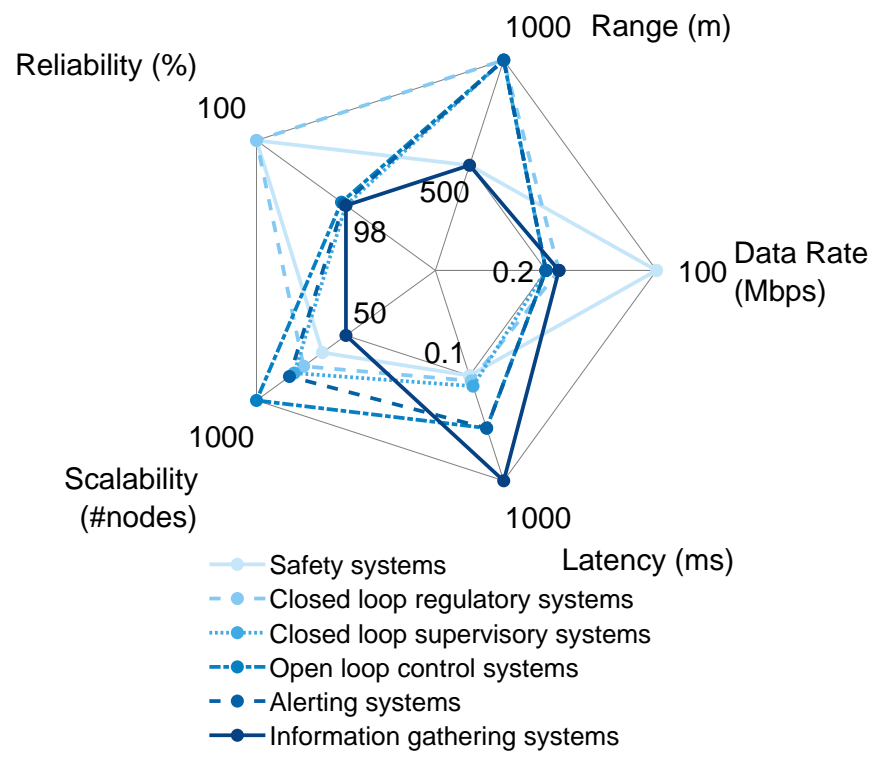

Figure 3. Different classes of industrial systems have significantly different performance requirements. 
Many of the stated requirements are interconnected and there is no single technology that covers all of them simultaneously. The inevitable trade-offs, their causes and consequences are elaborated in the next section.

\section{Trade-offs in Wireless Network Design}

Providing wireless communication to heterogeneous applications, including the time-critical ones, over a wide industrial site is a conflicting task. For example, 1 All three are partly determined by the choice of frequency band and bandwidth but also with other design choices that create additional interlocks between the performance parameters. These trade-offs, illustrated in Figure 4, complicate the design of wireless solutions.

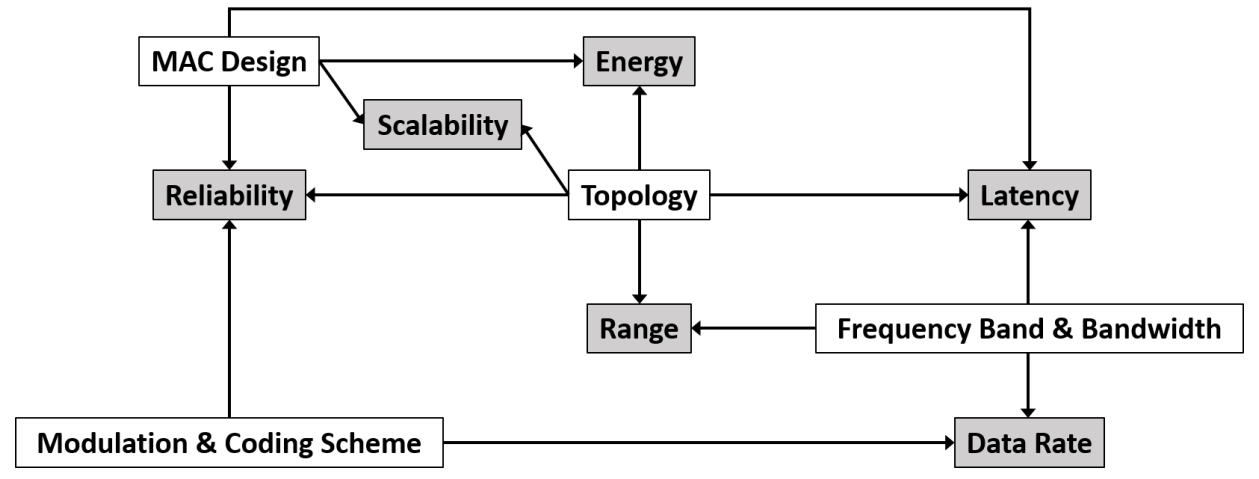

Figure 4. Network design choices (white rectangles) influence several performance properties simultaneously (grey rectangles), thus creating the trade-offs between them.

\subsection{The Transmission Range}

The transmission range is mainly determined by the transmission power, typically limited by regulations [11], the radio and propagation properties, as well as coding and modulation complexity. If a radio transmits at a constant power, lowering this complexity rate permits the correct decoding of a weaker or more distorted signal by a receiver, thus extending the transmission range. Also, higher frequency bands with more bandwidth available enable higher data rates and faster data transmission but they also have worse penetration capabilities which reduces the range in an industrial site full of obstacles. Range is largely determined by topology as well. Multi-hop topologies extend the range at the expense of latency, design complexity and energy consumption because of the need for synchronization of nodes, routing and so forth. In conclusion, low data rates at low frequencies and multi-hop topologies are prolonging the range but they all increase latency.

\subsection{Latency}

Latency is reduced by increasing the data rate, in turn enabled by more complex codings and larger bandwidths. Furthermore, multi-hop topologies increase the latency considering that forwarding and routing introduce additional delays. In addition, computing a new route when a link fails also introduces delay which can render multi-hop topologies useless in low-latency time-critical applications. Medium Access Control (MAC) design has a significant impact on latency as well, especially in IoT technologies where devices aim to sleep as long as possible to save energy, therefore delaying transmissions and receptions. MAC protocols can be classified into four classes: (1) Fixed Assignment Protocols where resources are divided among the nodes for a defined time duration, (2) Demand Assignment Protocols where resources are provided to a node on demand, (3) Random Access Protocols where resources are divided randomly and (4) Hybrid Protocols that combine fixed or demand assignment with random access. Fixed Assignment Protocols such as Time Division Multiple Access (TDMA) introduce determinism and achieve lower latency than random access protocols under very high load, but under low load they waste resources by inefficient usage of the channel time, 
where random access protocols achieve lower latency. Demand-based protocols are not suitable for low-latency time-critical communications given that explicitly asking for resources every time takes up bandwidth and adds up to latency. For heterogeneous industry applications, hybrid approaches are the most promising given that they aim to combine the benefits of both fixed assignment and random access protocols, while surpassing their limits at the same time and adapting to the network conditions. In addition, retransmissions need to be kept a minimum as they also increase the latency.

\subsection{Reliability}

Reliability is determined by topology, MAC design and Modulation and Coding Scheme (MCS). One of the major setbacks of wireless technologies in terms of reliability, in comparison to their wired counterparts, is the inter- and intra-technology interference on air which can cause collisions and increase packet loss. Technologies that operate in licensed bands reserve a part of the spectrum for themselves, mitigating the issue. However, spectrum is a scarce resource and it comes at a high price. Private deployments are not possible in reserved spectrum, disabling the possibility of local control over a network. Shared spectrum, on the other hand, can be shared by any number of technologies which can try and mitigate interference by channel hopping or using some MAC layer mechanisms such as Listen Before Talk (LBT). Furthermore, in a single-hop networks, the success probability is entirely dependent on a single link, opposed to multiple links in multi-hop networks. Reliability can be improved by employing both retransmissions and repetitions at the MAC layer, which also add to the latency. To reduce the number of retransmissions, error control techniques such as Forward Error Correction (FEC) can be used. Coding schemes and modulation largely define reliability. Coding rates create extra error checking bits that make modulation more reliable. Modulation schemes are more reliable as they have fewer points on the constellation diagram but also slower. That makes Binary Phase Shift Keying (BPSK) the slowest and the most reliable modulation compared to Quadrature Amplitude Modulation (QAM) and Quadrature Phase Shift Keying (QPSK), given that it only accommodates two points (one bit per burst). QPSK uses four constellations, whereas QAM can have any number of points. Any increase in the number of points on the constellation diagram reduces the space between them, leaving fewer margins for error. This makes QAM the fastest modulation scheme but more unreliable over longer distances.

\subsection{Data Rate}

Data rate is directly correlated with the available bandwidth and thus frequency band, on one hand, and with modulation and coding scheme on the other. More bandwidth enables higher data rates, while modulation techniques and coding schemes can further contribute to the achievable data rate by encoding more data into the signal. Unlicensed wireless technologies operate either in sub- $\mathrm{GHz}$ frequency bands $(400 \mathrm{MHz}, 800-900 \mathrm{MHz})$, in $2.4 \mathrm{GHz}$ or in $5 \mathrm{GHz}$. Sub-GHz technologies generally (although not universally) use narrower channels (few hundred kHZ) than those in GHz frequency bands (22 MHz Wi-Fi, $2 \mathrm{MHz} 802.15 .4$ ) and thus have more limited data rates than the $\mathrm{GHz}$ ones.

\subsection{Energy Consumption}

Energy consumption depends on data rate, topology and MAC design, as well as the hardware design of course. Low data rates result in long transmission times, which increases the energy consumption of the node and reduces the battery lifetime. Topology wise, nodes in multi-hop networks consume more energy than in single-hop networks given that, besides their own transmissions and receptions, they also need to forward other nodes' packets. Energy-efficiency of data forwarding paths give the routing protocols a strong influence over energy consumption as well. Complex coding and decoding operations also contribute to energy consumption. For example, FEC has been omitted in commercial 802.15.4 based networks due to the energy consumption of the decoding operation. Nevertheless, employing FEC could reduce the overall energy consumption as less energy would be 
spent on retransmissions and rescheduling [28]. Besides, MAC design has a significant impact on energy consumption as it defines scheduling and hence the radio on and off times.

\subsection{Scalability}

Scalability is primarily determined by MAC design. Scheduling, contention resolution and other MAC mechanisms work together to provide maximum network capacity. In single-hop networks, the network capacity upon reaching the upper limit can only be extended by deploying more base stations. However, in practice the density of such base stations is limited. Multi-hop networks address this issue by allowing for wireless data forwarding, at the expense of overall throughput. In TDMA-based protocols, network density is limited by the need for synchronization and time division in combination with QoS requirements.

\subsection{Spectrum Regulations}

Another tackling design choice is the one between unlicensed Industrial, Scientific and Medical (ISM) and licensed bands. On the one hand, worldwide permitted unlicensed operation reduces the runtime costs but has no regulatory protection against interference by other wireless networks operating in the same frequency band. On the other hand, even though licensed bands prevent interference, they typically depend on an external operator. Therefore, network issues cannot be immediately resolved on site, the external operator needs to resolve them. This introduces administrative delays which are unaffordable in time-critical industrial applications. Communication technologies operating in the unlicensed spectrum are maintained and managed locally. However, several co-located or overlapping wireless networks operating in the same frequency band will interfere with each other and can experience decreased QoS and extensive packet loss [14]. In an effort to alleviate coexistence issues in unlicensed spectrum, regulatory bodies have issued a number of norms such as a Clear Channel Assessment (CCA) check before each transmission by all devices, that is, a device has to sense if the channel is free by energy detection or other types of Detect And Avoid (DAA) mechanisms [14]. Although the DAA mechanisms improve the coexistence between the contending wireless nodes and networks, collisions can still occur. Apart from collisions, medium sensing adds to the latency and introduces non-determinism due to the medium congestion. Aforementioned facts significantly limit the use of wireless solutions in closed loop control applications in automation industry. A limiting regulation is present in unlicensed sub-GHz spectrum as well. Devices with an operating range of $863-868 \mathrm{MHz}$ in Europe, 916.5-927.5 MHz in Japan and 902-928 MHz in the US must comply with the maximum duty cycle limit of $2.8 \%$ and $10 \%$ for the, Access Point (AP) provided that they support LBT and Adaptive Frequency Agility (AFA) features, $1 \%$ otherwise [11].

\section{Wireless Technologies for Industrial Applications}

To support cyclic communication between sensors, actuators and controllers, sufficient throughput, latency and range is needed. This paper only considers wireless technologies that have the potential to enable real-time cyclic communication over a range comparable to the size of an industrial site, thus larger than a hundred meters. In line with that, we consider the promising IIoT technologies to be LoRa, IEEE 802.11ah and NB-IoT out of single-hop long range networks, WirelessHART, ISA100.11a, BLE and 802.15.4g/e physical (PHY) with 802.15.4e TSCH MAC out of long range multi-hop networks. The performance of each individual technology in terms of requirements presented in Section 2 and trade-offs presented in Section 3 is discussed below.

\subsection{Long Range Networks}

Single-hop long range networks that have the potential to enable real-time cyclic communication over a range comparable to the size of an industrial site are introduced in the remainder of this section. 


\subsubsection{LoRa}

LoRa is a proprietary wireless data communication technology which specifies a PHY layer only. A popular MAC for use with LoRa is the open LoRaWAN specification. LoRa PHY uses Semtech's proprietary Chirp Spread Spectrum (CSS) radio modulation to reduce receiver complexity while achieving long range. CSS is resistant to Doppler effects and multipath fading. A LoRa receiver can decode transmissions $\sim 20 \mathrm{~dB}$ below the noise floor, enabling very long communication distances while using very limited power. CSS is a spread spectrum technique where the signal is modulated by chirp pulses whose frequency linearly varies, parametrized by the orthogonal Spreading Factors (SFs), which can take values 7-12. The higher the SF, the longer packet transmission time and the more reliable its reception. Therefore, high SFs improve robustness against interference and counteract heavy multipath fading characteristic for indoor propagation and urban environments. This comes at the cost of low data rates and much higher energy consumption. Considering $125 \mathrm{kHz}$ channels, the data rates range from $0.25 \mathrm{kbps}$ to $5.47 \mathrm{kbps}$. These very low rates result in long transmission times (and medium usage) even for small packets. For example, a 17-byte sensor reading would take over $1.5 \mathrm{~s}$ to transmit at SF12 and cca. $70 \mathrm{~ms}$ at SF7. Combining the LoRaWAN MAC and LoRa PHY data rates results in an rather low network capacity per gateway of less than $0.02 \mathrm{MB}$ per hour [29].

An experimental study on the range of LoRaWAN [30] showed that it can achieve ranges up to $7.5 \mathrm{~km}$ using SF10 and packets with 10 bytes of payload, resulting in 0\% Packet Error Rate (PER) and $-126 \mathrm{dBm}$ Received Signal Strength Indication (RSSI). When using the specification of the Wireless M-Bus according to EM13757-4, 50 bytes of payload, Frequency Shift Keying (FSK) modulation with an FSK deviation value of $50 \mathrm{kHz}$ and a data rate of $100 \mathrm{kbps}$, LoRa achieves up to $1.35 \mathrm{~km}$ of range with $0 \%$ PER and up to $3.6 \mathrm{~km}$ of range for $<10 \%$ PER.

LoRaWAN is based on pure ALOHA, thus collisions pose the biggest issue in such networks given the long air-times. According to LoRaWAN, edge-devices' downloading needs determine their class:

- $\quad$ Class A devices have a single Receive Window (RW) scheduled immediately after a corresponding uplink connection,

- Class B devices can schedule additional RWs,

- Class C devices continuously listen and can receive almost anytime.

The more RWs, the more energy devices consume, that is, the power consumption increases over the classes A through C.

LoRaWAN supports both confirmed and unconfirmed messages. However, downlink capability of LoRaWAN networks is highly limited. With an increasing traffic load, RWs for sending acknowledgements (ACKs) to confirmed messages are more frequently missed as the gateway cannot transmit at the start of a RW due to the duty cycle restrictions. When a gateway sends an ACK in either RW1 or RW2, it aborts all ongoing receptions further decreasing the Packet Delivery Ratio (PDR) [31]. Currently, various scheduling solutions are being investigated that aim to improve LoRaWAN capacity and reliability, properties that are of interest to industrial IWSANs [32]. At this moment, even with the adaptation of LoRa/LoRaWAN to TSCH mechanism, this technology can only fulfill requirements for those industrial application scenarios classified to classes 3-6 that need cycle time in the order of seconds in the best case scenario, or even minutes for very dense deployments [33]. Reference [34] analyzed end-to-end latency of LoRaWAN in IIoT use cases and showed that LoRaWAN may be applicable for a subset of IIoT use cases where lower SFs can achieve end-to-end delay below $400 \mathrm{~ms}$. Long distance transmissions ranging up to $15 \mathrm{~km}$ require a high SF and result in the end-to-end latency well above one second, which is far beyond real-time availability of sensor data.

In conclusion, the LoRa PHY allows long range, but the low network capacity and current class A MAC design restricts use cases to those with a combination of low data requirements, a majority of uplink traffic and overall traffic volumes that remain far below the theoretical capacity. 


\subsubsection{IEEE 802.11ah/Wi-Fi HaLow}

IEEE 802.11ah, marketed as Wi-Fi HaLow, can serve up to 8192 stations per AP, a much higher value than the previous 802.11 iterations. In addition to the $1 \mathrm{~km}$ coverage, its relay functionality can further increase both the network size and coverage. To limit interference between stations and collisions, it introduces the Restricted Access Window (RAW) mechanism which combines the deterministic and the stochastic channel access. RAW restricts the channel access for a specified group of stations assigned to the time slots within the RAW. Stations assigned to specific slots within a RAW contend for the medium in their corresponding slots employing Enhanced Distributed Channel Access (EDCA)/Distributed Coordination Function (DCF). The stations are not allowed to contend for the medium in RAW slots they are not assigned to. RAW configuration and assignment is configurable and can change every beacon interval. RAW can reserve channel time for any group of stations. It improves throughput in dense IoT networks where many stations contend for the medium simultaneously. RAWs can contribute to introducing determinism in a network, if configured accordingly. However, an arbitrary RAW configuration can also degrade network performance if RAW is not configured with respect to the traffic patterns of the end devices. RAW duration can be configured to any value between $500 \mu$ s and the beacon interval.

IEEE 802.11ah demonstrates robustness in industrial environments given that it has better penetration capabilities than $2.4 \mathrm{GHz}$ technologies due to sub-GHz frequency bands. It employs Wi-Fi WPA3 for security and can be used for over-the-air software updates [35], besides monitoring and control.

The minimal feasible cycle time in a 802.11ah network equals 2 or 4 beacon intervals, given that 2 to 4 hops are needed to complete a single cycle in a control loop in a star network: two if the controller is wired to the AP and four otherwise. Each hop can only be executed in a single beacon interval. Beacon intervals can be reduced in order to support low-latency loops but reducing the beacon intervals makes the spectrum usage less efficient [36]. The minimal cycle time for a single $99.99 \%$ reliable control loop with a wired controller is $32 \mathrm{~ms}$ and it can operate alongside 75 sensors reporting 64-byte measurements every $1 \mathrm{~s}$. Longer cycles enable the reliable support of more control loops, for example, 4 control loops with $64 \mathrm{~ms}$ cycle time and 70 sensors reporting every $1 \mathrm{~s}$. IEEE 802.11ah can offer differentiated QoS to different types of end nodes in a dense deployments [37,38]. In addition to classes 3-6, this technology may be capable of providing QoS to class 2 as well, supporting low latency closed loops ( $<100 \mathrm{~ms})$ along with other non-critical traffic. However, the lower the latency requirement, the fewer nodes the network can support due to the used up bandwidth. Hence, there is a trade-off between the scalability and the latency that can be supported in practice. Given the lack of available hardware at the market at this time, it is yet to be seen how does this technology perform in the real world, outside of simulation studies.

\subsubsection{Narrowband-IoT}

NB-IoT aims to offer deployment flexibility allowing an operator to use only a small part of the available spectrum for the technology. It targets ultra-low-end IoT applications. NB-IoT supports three coverage classes, namely (1) normal, (2) robust and (3) extreme coverage class. Those correspond to the Minimum Coupling Losses (MCLs) of 144 dB, $158 \mathrm{~dB}$ and $164 \mathrm{~dB}$ respectively. In NB-IoT, the uplink latency consists of the system synchronization, broadcast information reading, random access, resource allocation, data transmission and feedback response [39]. In uplink, NB-IoT makes use of single- $(20 \mathrm{kbps})$ and multi-tone $(250 \mathrm{kbps})$ channels. A single-tone technology implies either 12 or 48 continuous sub-carriers with sub-carrier spacing of either $15 \mathrm{kHz}$ or $3.75 \mathrm{kHz}$, respectively. Multi-tone technology implies 12 continuous sub-carriers, with $15 \mathrm{kHz}$ spacing, grouped in 3, 6 or 12 continuous sub-carriers. Spacing of $3.75 \mathrm{kHz}$ results in higher coverage than $15 \mathrm{kHz}$ spacing due to the higher power spectral density, which makes the cell capacity $8 \%$ larger for $3.75 \mathrm{kHz}$-spacing [40]. In downlink, Orthogonal Frequency-Division Multiple-Access (OFDMA) is employed with sub-carrier spacing of $15 \mathrm{kHz}$. 
Recent measurements on an actual public NB-IoT network showed that the achievable application layer throughput is significantly lower, at around 10 and $15 \mathrm{kbps}$ for uplink and downlink, respectively [41]. In terms of latency, a one-way latency of about $50 \mathrm{~ms}$ could be achieved under perfect channel conditions for small packets of 8 bytes. However, for deep indoor scenarios, latency increased to around $16 \mathrm{~s}$ for uplink and $8 \mathrm{~s}$ for downlink transmissions. Another experimental evaluation of NB-IoT using two different commercial platforms in a public NB-IoT network has observed the inconsistency in performance metrics, namely the energy consumption and latency [42]. The variability of energy consumption results in an imprecise predictability of battery life and causes a difference in performance of similar devices. Although NB-IoT is designed for delay-insensitive applications, some cases cannot tolerate the variability of latency amounting to tens of seconds or even minutes. Guaranteed reliability in NB-IoT comes at a cost of variability [42].

NB-IoT is superior than most of the competition in terms of range, security and availability. However, its unpredictable latencies that can be in the order of seconds make it applicable for latency insensitive processes only. Its random resource reservation procedures make the connection latency high in dense networks. When connected, its throughput and latency can significantly vary depending on channel conditions due to the repetitions and changes in MCSes. Even though the hardware is inexpensive, NB-IoT comes with a subscription fee, while all other technologies listed in Tables 2 and 3 can be deployed privately. In conclusion, it is significantly more robust than competition which makes it suitable for class 2 latency insensitive applications. It can also be used in classes 3-6.

\subsection{Long Range Multi-hop Networks}

Multi-hop networks not only extend the range but also substantially contribute to the reliability as in mesh networks there are (typically) 2 or more paths from the source to the destination, meaning that the loss of a link will not result in a communication failure like in single-hop networks. However, nodes in multi-hop networks consume more energy because aside from their own packets, they also must transmit and receive (forward) other nodes' packets. Routing the packets adds up to latency and complexity. Thus, even though the scalability of multi-hop networks is not explicitly limited, deployments typically do not include more than 5 hops. The theoretical limit on the maximal network density is based on the addressing space, which for most of the analyzed technologies is $2^{32}$, thus considered unlimited. However, the practical capacity limit is determined by the network latency and individual device's energy consumption. They both increase with the number of the communication links in the network. In addition, bottlenecks can occur at one or more devices that communicate directly with the gateway in a mesh network. Because of this, data traffic can significantly increase resulting in the increase of the devices' energy consumption. Also, the maximum achievable update rate is greatly influenced by the network density given that high update rates generate more traffic than low update rates.

\subsubsection{WirelessHART}

Along with the PHY, MAC, network and transport layer, the WirelessHART protocol stack also includes the application layer. The application layer is HART, which is compatible with existing wired HART solutions.

Both WirelessHART and ISA100.11a combine TDMA and frequency hopping at the MAC layer. To access the channel, WirelessHART uses a two-dimensional matrix consisting of time slots and 15 available channels. Time slots are grouped in superframes which periodically repeat throughout the entire network lifetime. Variable-length superframes are also supported and at least one superframe must be enabled. Superframes can be added or removed during the operation of the network and they are managed by the network/system manager. Time slot duration is fixed to $10 \mathrm{~ms}$ in WirelessHART. This scheme allows multiple devices to transmit data at the same time using different channels. However, a single device can only make use of a single channel per time slot. WirelessHART's (single) time slotted channel hopping mechanism also identifies noisy channels and blacklists them, 
thereby greatly improving the network reliability. In addition, this technology can achieve up to 99.999\% reliability by supporting mesh topologies [43]. Security in WirelessHART communication is provided by the 128-bit Advanced Encryption Standard (AES) authentication.

WirelessHART is the oldest and the most experimentally evaluated wireless solution for IIoT [44-47]. A study has demonstrated control over WirelessHART with the update rate of $20 \mathrm{~ms}$ [45]. However, this and similar studies evaluate the simplest variant of a WirelessHART network comprising of a sensor, an actuator, an AP, a gateway (GW) and a network manager. Such a low update rate is achievable only in star topologies and with very short superframes that disable scaling the network up. Even though not more than 5 hops are advised in industrial networks, scaling WirelessHART is possible (1) using multiple APs and (2) using several WirelessHART gateways connected to a HART-over-IP backbone. This is very much needed in dense industrial environments as only 8 devices with $500 \mathrm{~ms}$ reporting period can be supported by a single AP [48]. In practice, a single WirelessHART AP can support 25,50, 80 or 100 devices at $1 \mathrm{~s}, 2 \mathrm{~s}, 4 \mathrm{~s}$ and $8 \mathrm{~s}$ reporting period respectively. Both bidirectional (cyclic) traffic and an increment in hops doubles the latency or halves the number of nodes. Hence, WirelessHART is eligible for use in classes 2-6 of industrial systems, although it is limited either by latency or by scale.

\subsubsection{ISA100.11a}

The ISA100.11a protocol is designed for secure and reliable wireless operation for noncritical monitoring, alerting, supervisory control, open- and closed-loop control applications (classes 2-6). Although it is in many aspects similar to WirelessHART, unlike WirelessHART it provides flexibility for customizing the operation of a system. It is based on 802.15.4 PHY and achieves a single-hop range up to $100 \mathrm{~m}$. The ISA100.11a utilizes TDMA and Carrier Sense Multiple Access with Collision Avoidance (CSMA/CA) on MAC layer, as well as graph routing and channel hopping which improves reliability by avoiding busy channels. The CSMA/CA mode is commonly used for retries, association requests, exception reporting and burst traffic. The use of any single channel is reduced by the time-synchronized slots and channel hopping, resulting in the improvement of ISA100.11a's coexistence with other networks in the shared spectrum. Communication takes place in time-slots with configurable constant lengths, varying from $10 \mathrm{~ms}$ to $12 \mathrm{~ms}$. Time-slots are grouped in a superframe that periodically repeats in time. The length of the superframe is configurable and can differ for each node. Generally, long-period superframes result in high latency and low bandwidth. However, they conserve energy and contribute to the outspread allocation of digital bandwidth. Three methods are employed for limiting the use of undesirable radio channels and reducing the interference with other wireless networks: (1) CCA, (2) spectrum management and (3) and adaptive channel hopping. There are 15 (16 if the optional channel 26 is enabled) available channels to chose from in each time-slot. Three channel hopping sequences are defined, along with five hopping patterns.

In dense deployments, ISA100.11a devices can join the network faster than WirelessHART devices, as shown in Reference [49]. The WirelessHART network manager allows the initiation of the join process for each transmission opportunity (TXOP) and allocates the resources for publishing data during the join process. On the other hand, ISA100.11a employs dedicated advertisement links for join process initiation. At the network startup, the ISA100.11a gateway acts as a join proxy. However, each joined device may act as a join proxy, thus allowing more join opportunities. This results in faster joining of ISA100.11a devices in a dense deployment but not when there are only few devices. Reference [49] observed ISA100.11a to be slightly more reliable than WirelessHART, but also to have larger latency than WirelessHART in a cyclic communication.

Unlike WirelessHART, not all devices in ISA100.11a network must have routing capability. Without it, devices must be within one hop of a routing-capable device or the gateway. This significantly constrains the network's re-routing capability in case of link fading due to changes or movements in the plant, making the network less adaptable to changes. In addition, even though the standard does not impose a limit on the number of nodes, in practice it is only possible to connect up to 10 devices 
reporting every $0.5 \mathrm{~s}$ to a single AP, 25 devices reporting every $1 \mathrm{~s}, 50$ at $2 \mathrm{~s}, 80$ at $4 \mathrm{~s}$ or 100 at $10 \mathrm{~s}$ [48]. Both bidirectional (cyclic) traffic and an increment in hops doubles the latency or halves the number of nodes. This makes ISA100.11a suitable for application to classes 3-6, as well as to class 2 assuming the specific requirements of a class 2 application are in line with the latency and scale constrains of this technology.

\subsubsection{Bluetooth Low Energy}

Unlike classic Bluetooth which was designed as a point to point wire replacement, BLE provides increased range with $1 \mathrm{Mbps}$ data rates and recently introduced meshing. An experimental study [50] observed the range of around $50 \mathrm{~m}$ indoors. The observed outdoors range for non line of sight scenarios was $123 \mathrm{~m}$ and $165 \mathrm{~m}$ for $0 \mathrm{dBm}$ and $9 \mathrm{dBm}$ transmission gain respectively, versus $490 \mathrm{~m}$ and $790 \mathrm{~m}$ in line of sight scenarios. Instead of Bluetooth's 1-MHz wide 79 channels, BLE uses 40 channels of $2 \mathrm{MHz}$. It employs frequency hopping over 37 channels for (bidirectional) communication and 3 for (unidirectional) advertising. Two MAC layer roles are defined for the devices in BLE, master and slave. The master coordinates the medium access using a TDMA-based polling mechanism, in which it periodically polls the slaves in their corresponding Connection Events (CES) which are uniformly spaced within a configurable periodic interval called a Connection Interval (CI). Frequency hopping is employed in a way that each CE uses a different channel. The standard defines the CI duration as a multiple of $1.25 \mathrm{~ms}$ in the range from $7.5 \mathrm{~ms}$ to $4 \mathrm{~s}$. Therefore, the minimal cycle time equals the CI in a BLE control loop. Considering that the sample/update rate in an industrial system should be 3-4 times faster than the process time constant for condition monitoring and open loop control and 4-10 times faster for regulatory closed loop control, the minimal cycle times achievable with BLE increase to $22.5 \mathrm{~ms}-30 \mathrm{~ms}$ for non-critical traffic and $30 \mathrm{~ms}-75 \mathrm{~ms}$ for regulatory loops. BLE is theoretically capable of supporting 6 control loops exchanging 47-byte (max. size) packets with the minimal CI. Extending the CI for $n$ time units of 1.25 ms results in $S$ control loops, as follows:

$$
S=\left\lfloor\frac{7.5 \mathrm{~ms}+n \cdot 1.25 \mathrm{~ms}+T_{G B}}{2 \cdot 0.376 \mathrm{~ms}+0.15 \mathrm{~ms}+T_{G B}}\right\rfloor
$$

where $0.376 \mathrm{~ms}$ is the transmission duration of a 47-byte packet at $1 \mathrm{Mbps}, 0.15 \mathrm{~ms}$ stands for an Inter-Frame Spacing (IFS) and $T_{G B}$ (in ms) for implementation-specific guard band between two successive CEs. This reasoning is based on the one-to-one mapping between control loops and CEs in a single CI. Besides $2 S$ transmissions in a CI, we must fit $S$ IFSs between successive transmissions in CEs and $\mathrm{S}-1$ guard bands between CEs as well.

Bluetooth 5 provides the options to double the speed to $2 \mathrm{Mbit} / \mathrm{s}$ at the expense of range or up to fourfold the range at the expense of data rate and eightfold the data broadcasting capacity of transmissions by increasing the packet lengths. However, BLE's limited range makes it unsuitable for large scale IIoT deployments, as over 1000 basestations would have to be deployed to cover the same area as a single Wi-Fi HaLow basestation. To overcome this issue, Bluetooth introduced meshing. Communication in the Bluetooth Mesh standard in implemented using BLE advertising and scanning on the 3 advertisement channels. The standard uses a flooding mechanism where each node in the network repeats incoming messages, relaying them until the destination. Unlike in normal BLE advertising, Bluetooth Mesh transmissions are not scheduled based on an advertising interval. Instead, the nodes transmit after a random backoff time. In a Bluetooth Mesh network, increasing the number of relaying neighbors from 1 to 10 reduces the 2-hop round-trip time (RTT) from $47 \mathrm{~ms}$ to $33 \mathrm{~ms}$ for 41-byte packets and $10 \mathrm{~ms}$ scanning interval [51]. Increasing the number of hops from 1 to 4 increases the RTT from $22 \mathrm{~ms} 88 \mathrm{~ms}$. This is because the latency is influenced the most by the random backoff mechanism. However, the absence of a random backoff would increase the collision probability, decreasing the reliability and scalability of Bluetooth Mesh networks which makes it suitable only for classes 4-6 of industrial applications. 


\subsubsection{Time Slotted Channel Hopping}

A MAC layer with TSCH can operate on top of both $2.4 \mathrm{GHz}$ - and sub-GHz 802.15.4-based PHY. Assuming up to 4 hops, 802.15.4 networks that operate in unlicensed $2.4 \mathrm{GHz}$ frequency bands can achieve a range of up to $200 \mathrm{~m}$. On the other hand, their sub-GHz counterparts based on $802.15 .4 \mathrm{~g}$ can reach up to $1 \mathrm{~km}$ single-hop range [52].

In TSCH mode, time is slotted and time slots are grouped in slot frames. Both the time-slot duration and the number of slots in the slot frame are configurable. Slot duration is determined by the time needed to transmit a packet and receive an ACK and typically amounts $6 \mathrm{~ms}-10 \mathrm{~ms}$. The length of the slot frame is based on the trade-off between energy consumption and throughput. Decreasing the slot frames increases both the energy consumption and the throughput and vice versa. For cyclic communication in a closed loop, a node would need two slots in a slot frame. The cycle could repeat every slot frame, thus the cycle time trades off with the number of nodes in the network. A network of two nodes could exchange packets with $\sim 20 \mathrm{~ms}$ cycle time but 25 closed loops would increase the minimal cycle time to $500 \mathrm{~ms}$. Considering the best practices regarding the sampling in industrial communications, the minimal cycle times achievable with TSCH in practice increase to $60 \mathrm{~ms}-80 \mathrm{~ms}$ for non-critical traffic and $80 \mathrm{~ms}-200 \mathrm{~ms}$ for a single regulatory loop ( $2 \mathrm{~s}-5 \mathrm{~s}$ for 25 loops).

Besides time slotting, TSCH also utilizes channel hopping to diminish the impact of interference and channel fading. Nodes communicate with each other over 16 orthogonal channels and retransmit packets on different channels if initial attempts fail, thereby increasing reliability. Channel hopping scheme could benefit the scalability and the aggregate throughput of the network if all channels were used in a same time slot. However, this benefit is largely limited in industrial networks given that all slave nodes communicate with the same master node in the network, as illustrated in Figure 1. Unless a master node is able to send and receive on multiple channels simultaneously, which is not the default capability, nodes will be forced not to transmit simultaneously, regardless of the channel hopping scheme.

Furthermore, reliability of $2.4 \mathrm{GHz}$ TSCH networks may be significantly reduced in the presence of interference of co-located Wi-Fi networks, as shown in Reference [53]. When only a few channels in the TSCH matrix are affected by interference, only an increase of the transmission latency due to retransmissions could be expected. However in reality, the probability of eventual packet dropout is no longer negligible because of the quite low default retry limit (4) and the fact that one Wi-Fi channel overlaps many IEEE 802.15.4 channels (four to five). As Reference [53] shows, this results in an increase of packet losses, in turn reducing the reliability. Reliability could be increased by increasing the retry limit, hence trading off the latency and power consumption.

In conclusion, TSCH networks utilize the schedule on each node to achieve determinism and robustness against channel fading, as well as to conserve energy. That robustness and determinism makes TSCH suitable for class 2-6 industrial applications, assuming it can comply with the range requirement. However, there is a strong trade-off between latency and scalability, given that multiple channels cannot be used in parallel by different slave nodes due to the centralized master node.

\section{Discussions}

Key specifications of each technology introduced in Section 4 are listed in Tables 2 and 3. Note that not all numbers in Tables 2 and 3 can be taken for granted as they do not depict the trade-offs, but only present theoretical limits. NB-IoT operates in licensed spectrum. It can reach as far as its cellular infrastructure goes, and it is inherently more reliable than technologies that operate in shared spectrum. LoRa, IEEE 802.11ah and 802.15.4g operate in unlicensed sub-GHz frequency bands, which makes their long range a feature of their physical layers. LoRa operates in $863-870 \mathrm{MHz}$ frequency band in Europe, offering three sub-bands at $864 \mathrm{MHz}, 867 \mathrm{MHz}$ and $868 \mathrm{MHz}$. Five $125 \mathrm{kHz}$ channels are defined in $867 \mathrm{MHz}$ band and three in $864 \mathrm{MHz}$ and $868 \mathrm{MHz}$ band [54,55]. The three default channels in $868 \mathrm{MHz}$ band are to be implemented by every node, whereas the rest are optional. Wi-Fi HaLow operates in 863-868 MHz band in Europe and defines five 1-MHz channels and two 2-MHz 
channels [56]. Considering this, it is clear that Wi-Fi HaLow and LoRa's optional channels overlap in 3 out of 7 Wi-Fi HaLow channels and do not interfere with each other otherwise, which makes their parallel deployments possible. However, Wi-Fi HaLow can severely interfere with 802.15.4g [57], given that they operate in the same bands. The $2.4 \mathrm{GHz}$ unlicensed frequency band is widely utilized today and the technologies that operate in this band must pay special attention to coexistence. WirelessHART, ISA100.11a and 802.15.4e TSCH are all based on the 802.15.4 2.4 GHz PHY layer that operates in sixteen $2 \mathrm{MHz}$-wide and $5 \mathrm{MHz}$-spaced channels in $2.4 \mathrm{GHz}$ frequency band. They all employ frequency-hopping to improve the reliability of their transmissions, as they make use of exactly the same spectrum. Given their shorter single-hop ranges, these technologies employ multi-hop topologies to extend their range.

Table 2. Technical properties (top) and key performance indicators (bottom) of sub-GHz wireless technologies for the IIoT domain.

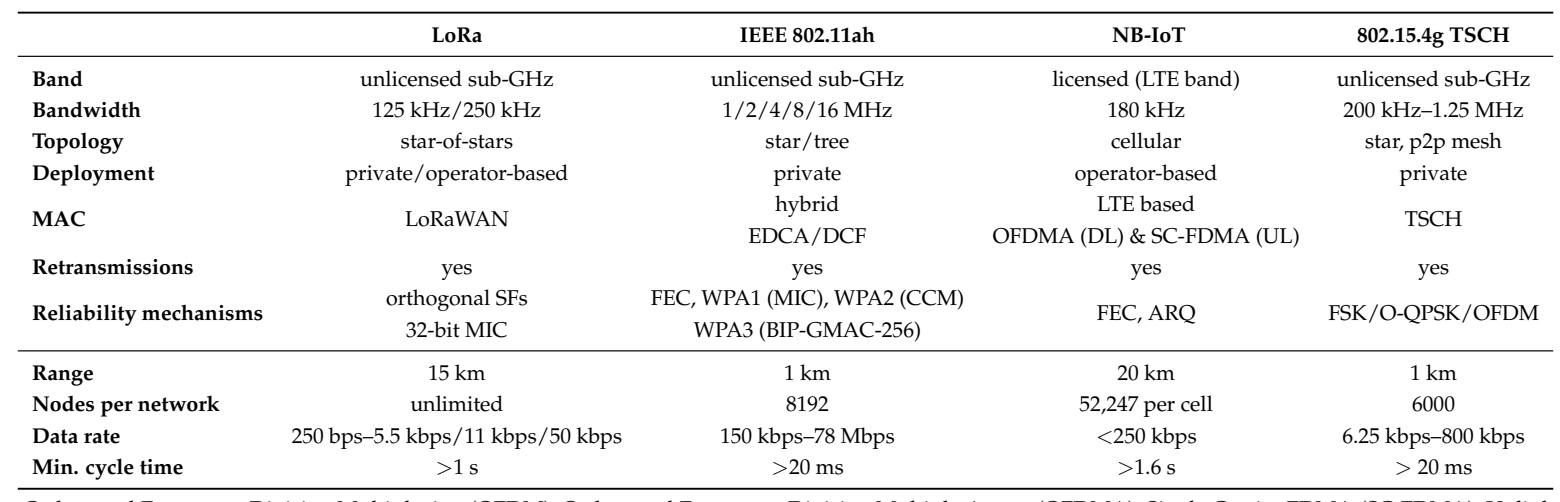

Orthogonal Frequency-Division Multiplexing (OFDM); Orthogonal Frequency-Division Multiple Access (OFDMA); Single Carrier FDMA (SC-FDMA); Uplink (UL); Downlink (DL); Automatic Repeat reQuest (ARQ); Offset QPSK (OQPSK); Counter Mode Cipher Block Chaining Message Authentication Code (CCM); Broadcast/Multicast Integrity Protocol (BIP); Galois Message Authentication Code (GMAC); Message Integrity Code (MIC).

Table 3. Technical properties (top) and key performance indicators (bottom) of IIoT wireless technologies based on 802.15.4 2.4 GHz PHY layer and BLE.

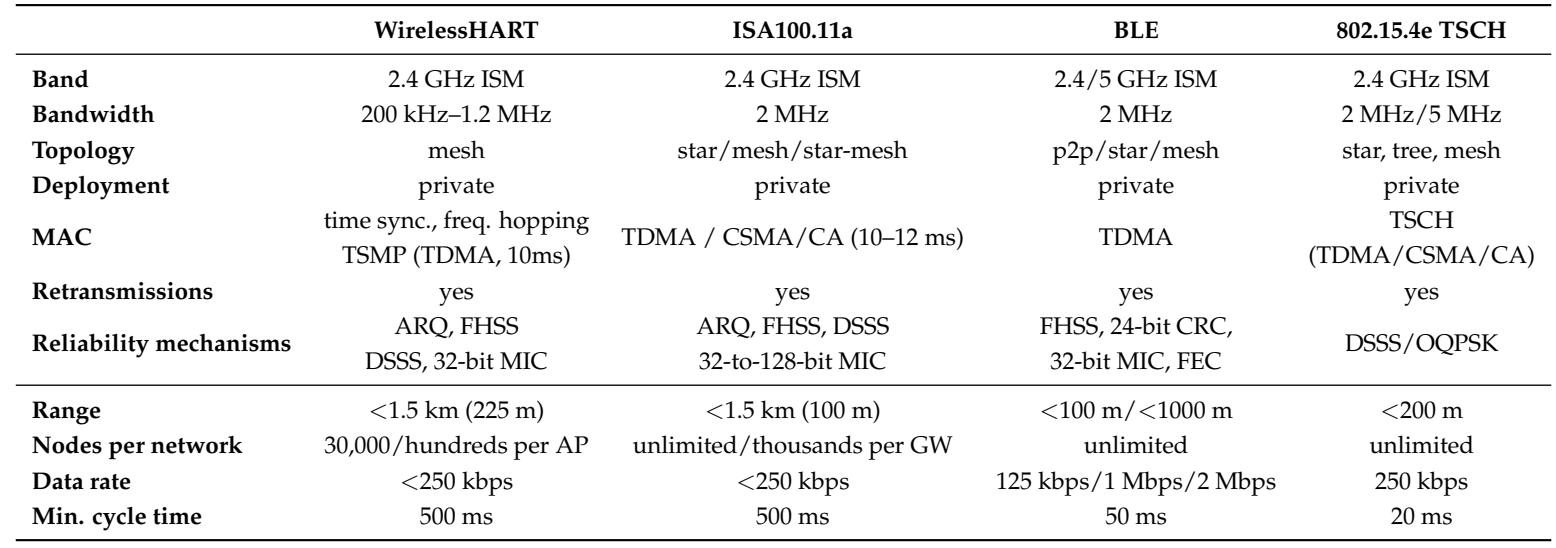

Automatic Repeat reQuest (ARQ); Frequency-Hopping Spread Spectrum (FHSS); Direct Sequence Spread Spectrum (DSSS); Gateway (GW); Offset QPSK (OQPSK); Message Integrity Code (MIC).

Wireless networks have the benefit of mitigating cabling for communication but the question of power cables still remains. Ideally, power cables could also be mitigated when the devices can live long enough drawing the power from the batteries available today. This is feasible for sufficiently large cycle times. Therefore, it is important to also consider the energy consumption. To evaluate the possibility of entirely wireless deployments that do not need power cables, an experimental energy efficiency comparison between LoRa, NB-IoT, IEEE 802.11ah and IEEE 802.15.4g (Wi-SUN with TSCH) was performed. The experiments combine energy consumption values of state-of-the-art off-the-shelf radios obtained from their data sheet (cf. Table 4), with simulated performance analysis. At the 
time of this study, no off-the-shelf radio was available for Wi-Fi HaLow and the same radio was assumed as for $802.15 .4 \mathrm{~g}$ (Atmel AT86RF215), as it supports the required modulation and coding schemes. The simulation experiments were performed using the ns-3 network simulator for LoRa [58], NB-IoT [59] and Wi-Fi HaLow [60]. Using the 6TiSCH simulator, 802.15.4g was evaluated [61]. A single device and a single gateway/AP is considered in the simulations. Hence, the results do not take into account scalability or contention. To maintain simplicity, predictability and comparability of the results, the experiments did not take into account any packet loss due to propagation errors, interference or collisions. As such, the PHY and channel contribution to the energy consumption is limited to the time-on-air of the radio for the reported packet sizes (including preamble and PHY/MAC header). The main contribution of the results pertains to the energy consumption of the MAC layer protocols of the considered technologies. For this reason, we used a simplified linear battery discharging model.

Table 4. Technology-specific parameters for energy consumption simulations.

\begin{tabular}{|c|c|c|c|c|}
\hline & IEEE 802.15.4g & IEEE 802.11ah & LoRaWAN & NB-IoT \\
\hline TX power $(\mathrm{dBm})$ & \multicolumn{2}{|c|}{14} & 23 & 20 \\
\hline $\begin{array}{c}\text { Technology-specific } \\
\text { parameters }\end{array}$ & $\begin{array}{l}113 \text { slots @ } 40 \text { ms per frame } \\
\text { 2-FSK-100 kHz (50 kbps) } \\
0.33 \% \text { DIO and EB probability }\end{array}$ & $\begin{array}{c}4096 \mathrm{~ms} \text { beacon interval } \\
\text { MCS } 10-1 \mathrm{MHz}(150 \mathrm{kbps}) \\
1 \text { RAW group, } 1 \text { slot }\end{array}$ & $\begin{array}{l}\text { no repetitions } \\
\text { no ACK }\end{array}$ & $\begin{array}{c}\text { RRC: } 10 \mathrm{~s}, \mathrm{DRX}: 0 \mathrm{~s}, \\
\text { PSM: TI s } \\
\text { no repetitions }\end{array}$ \\
\hline Microcontroller & \multicolumn{4}{|c|}{ ARM Cortex M3 @ 32 MHz (3.38 mA power consumption) } \\
\hline
\end{tabular}

Frequency Shift Keying (FSK); Enhanced Beacon (EB); DODAG Information Object (DIO); Destination Oriented Directed Acyclic Graph (DODAG); Modulation and Coding Scheme (MCS); Restricted Access Window (RAW); Radio Resource Control (RRC); Power Saving Mode (PSM); Transmission Interval (TI); Discontinuous Reception (DRX)

Wi-Fi HaLow is significantly more energy efficient than $802.15 .4 \mathrm{~g}$ with TSCH. This improvement is due to the higher supported data rate that leads to shorter transmission times. This result is also reflected in the predicted battery lifetime shown in Figure 5. Given a sufficiently large battery of $2000 \mathrm{mAh}$, the lifetime of Wi-Fi HaLow is expected to be above 10 years for a 10-min transmission interval, without battery replacement. For $802.15 .4 \mathrm{~g}$, battery life expectancy is under 3 years in the same scenario. For the long-range contenders, it is under a year, as shown in Figure $6 b$, except the LoRaWAN SF7 that can live up to 7.5 years (cf. Figure 6a). The compared configurations of 802.15.4g and 802.11ah do not represent the best case scenario regarding energy consumption, as both technologies are configured to use low data rates. However, the chosen settings result in similar coverage range. In a line-of-sight scenario, 802.15.4g FSK-50 reaches a good PDR up to $700 \mathrm{~m}$, while FSK-200 goes up to $420 \mathrm{~m}$ [62]. For 802.11ah [63], MCS10 (150 kbps) has a range of $700 \mathrm{~m}$ to more than $1000 \mathrm{~m}$. For a higher data rate, $802.15 .4 \mathrm{~g}$ will achieve better energy efficiency but it's achievable range would be very different from that of MCS10 of 802.11ah. Similarly, 802.11ah will achieve better energy efficiency for higher data rates (MCS10 represents the lowest possible data rate and thus the worst case).

Figure 6 illustrates the benefits of low power modes in the design of a technology. As shown in the Figure, the lifetime of LoRaWAN devices is much higher than that of NB-IoT. Power consumption of LoRaWAN devices in transmission (TX)/reception (RX) mode is the dominant factor of the overall consumption, considering the very low power consumption in the idle/sleep mode (cf. Table 4). This results in a large difference in lifetime between LoRaWAN devices that use SF7 and SF12. The difference between SF7 and SF12 is especially large when the transmissions are frequent. The rarer the transmissions, the smaller the difference between SF7 and SF12 as idle/sleep time prevails and the $\mathrm{TX} / \mathrm{RX}$ time becomes less significant in comparison to the idle/sleep time.

The larger difference in battery lifetime between NB-IoT MCS9 and MCS4 occurs due to the better efficiency of MCS9 which reflects not only in data TX/RX but also in signaling between the transmissions which adds up to the difference. The difference between MCS9 and MCS4 also reduces as the traffic interval increases, similarly as the difference between spreading factors of LoRaWAN. 
NB-IoT has higher data rate than LoRaWAN, thus it is more efficient in terms of TX/RX. However, the NB-IoT hardware is less efficient in terms of energy consumption in all four modes, so LoRaWAN becomes more energy efficient relative to NB-IoT. Higher data rates of NB-IoT cannot compensate for the energy efficiency of LoRaWAN in the evaluated scenarios.

It is important to note that the shelf life also affects the battery life. The lifetimes illustrated in Figures 5 and 6 represent the ideal cases which only take into account the consumption of a radio and a microcontroller. Hence, the presented results do not take into account energy consumption of peripherals, self-discharge of battery nor other power drains. For example, when considering only radio and microcontroller, NB-IoT platform using MCS4 and MCS9 would need around $230 \mathrm{mAh}$ and $155 \mathrm{mAh}$ respectively to run for three years. For the same time period, LoRaWAN radio and microcontroller using SF7 and SF12 would consume $8 \mathrm{mAh}$ and $47 \mathrm{mAh}$, when ignoring other power drains and employing sleep mode (45 mAh and $83 \mathrm{mAh}$ with idle mode). However, the above mentioned factors need to be taken into account when estimating very long battery lives as they make the largest part of energy consumption over a long time.

This paper focused mostly on PHY and MAC layer features of the considered wireless technologies, even though some technologies also address commissioning, security, roaming and other higher layer features. In addition to PHY and MAC, LoRaWAN defines a complete network architecture, various device types, commissioning and security (network and application). Parameter configurations and network management are generally implementer specific for all the considered technologies. LoRaWAN is configurable in terms of many parameters such as:

- $\quad$ spreading factor (and thus data rate): fixed choice or adaptive,

- reliability: ACKs or multiple transmissions of the same packet without downlink ACKs,

- $\quad$ higher layer logic: raw payload or Internet Protocol (IP) compliant stack.

Choosing the network setup for a certain scenario is not always straightforward, which motivated using optimization techniques to choose the optimal parameters [64]. Moreover, LoRaWAN does not impose the coordination of transmissions of class A devices, which might impact scalability in real deployments. Similarly, Wi-Fi HaLow defines PHY and MAC layer, as well as layer 2 security upon connecting. On top, IP compliant stack is to be used. Wi-Fi HaLow is highly configurable, defining a multitude of different parameters to be configured such as MCS, RAW, Traffic Indication Map (TIM) and others $[37,65,66]$. The configurable parameters can significantly influence the performance and the lifetime of the network. Higher layers of NB-IoT can be both IP- and non-IP compliant. NB-IoT also defines some configurable parameters such as extended Discontinuous Reception (eDRX) and PSM timers. However, other settings are under control of the network operator. The standard IEEE 802.15.4g/e TSCH defines PHY and MAC, with TSCH MAC layer specifying how to execute the schedule but not how to define it. Given that a schedule significantly impacts the network performance, there are standardization efforts in Internet Engineering Task Force (IETF) on scheduling functions but there is also a choice between centralized and decentralized scheduling. WirelessHART and ISA100.11a share a similar concept as TSCH. They have self-configuration capabilities greatly simplifying the deployment. They define the full stack and are centrally managed by the network manager that makes use of commands defined by the standard for network management [67]. They are out there for a long while and are quite well understood. BLE also defines the entire stack as well as other aspects such as commissioning. BLE has configurable parameters such as advertisement interval and connection interval that influence its performance. Different combinations of the configurable parameters can result in a different performance of any technology in various scenarios, thus the interdependence of the configurable parameters needs to be empirically examined. 


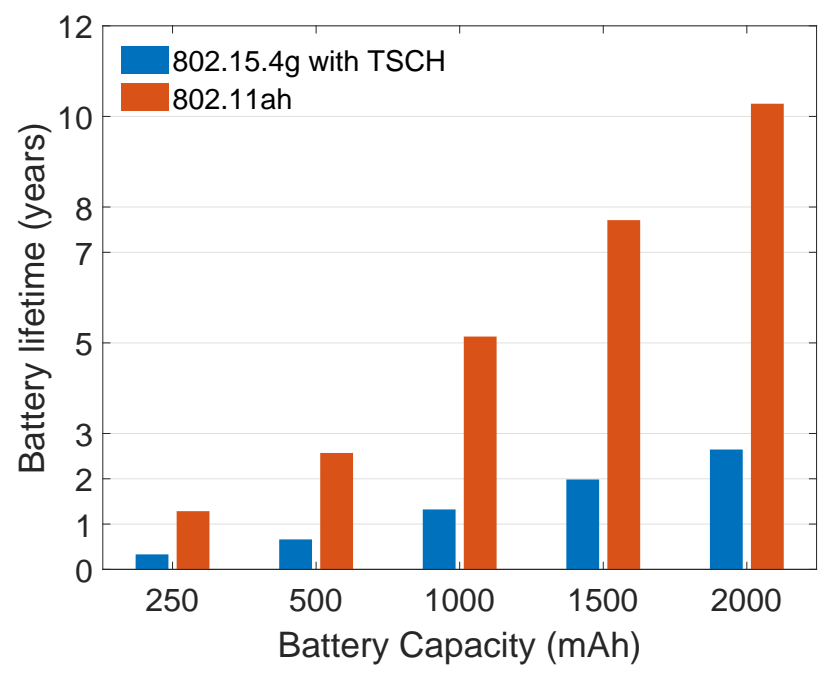

Figure 5. Battery lifetime of a device (both radio and microcontroller) transmitting once every $10 \mathrm{~min}$ for mid-range technologies (>1 km).

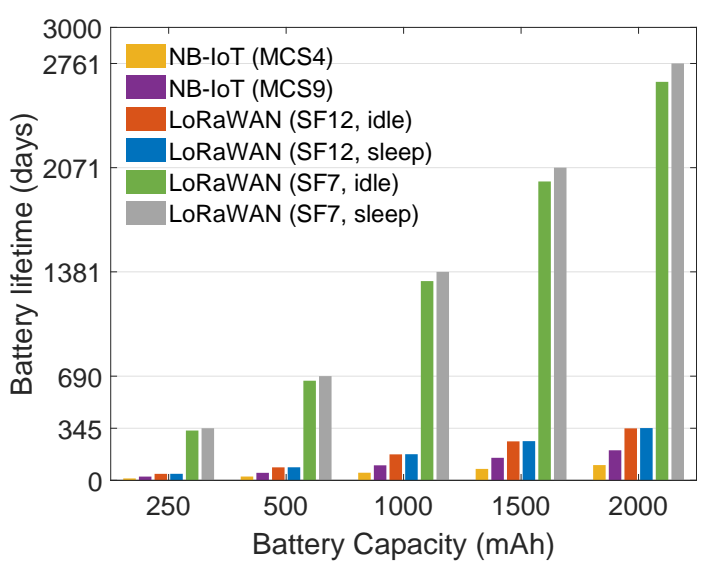

(a)

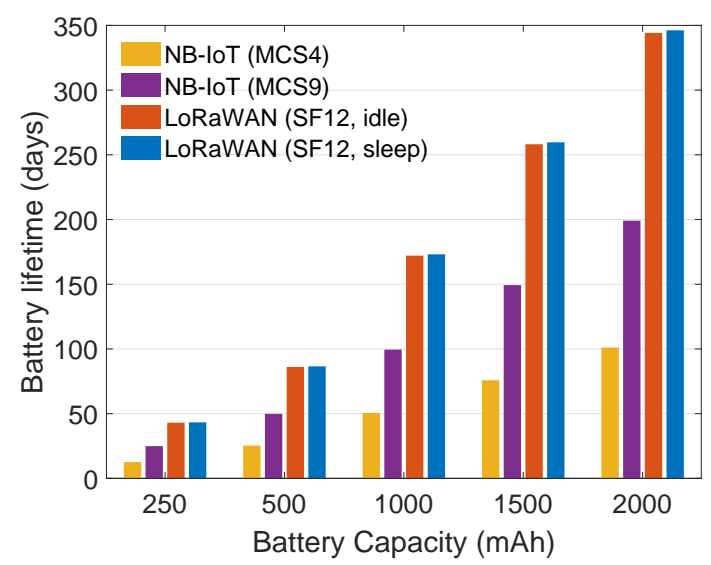

(b)

Figure 6. Battery lifetime of a device (both radio and microcontroller) transmitting once every $10 \mathrm{~min}$ for long-range technologies $(<10 \mathrm{~km})$. (b) is a subset of (a) where LoRaWAN SF7 is omitted for clarity.

\section{Conclusions}

LoRa, IEEE 802.11ah, NB-IoT, BLE, WirelessHART, ISA100.11a and TSCH can provide site-wide network coverage if configured and deployed accordingly, at the cost of data rate and/or latency and/or scalability. The trade-offs of the evaluated technologies are basically equivalent qualitatively but greatly vary in quantity, as elaborated in Section 4. NB-IoT and LoRa have potential to serve non-critical class 4-6 systems. On top, NB-IoT is more suitable for frequent downlink traffic because of LoRaWAN's downlink limitations, which makes it eligible for class 3 systems, as well as latency insensitive class class 2 systems (regulating very slow processes). However, NB-IoT typically comes with a subscription fee and due to the operator involvement, maintenance is slower. Thus, both have pros and cons, as well as similarities. BLE could serve supervising non-critical systems given its inherent unreliability in mesh topology and range limitations in star. TSCH, on the other hand, provides necessary reliability for critical services but its scale and latency are limited by the typical industrial fieldbus topology. WirelessHART, 802.15.4e TSCH and ISA100.11a are similar in terms of both reliability and latency. They all operate in the same frequency band and they all employ channel hopping to improve coexistence. Although they provide high data rates in comparison to other technologies, mesh topology increases the latency and limits the scalability. Still, these technologies 
are being deployed in class 2-6 systems. Finally, Wi-Fi HaLow trades data rate for range and latency but can still support the lowest latencies compared to reviewed technologies in networks of limited scale. Its star topology and novel MAC design contribute to high energy efficiency. Other technologies exhibit various trade-offs as well, ergo the choice of appropriate technology for a certain application is very challenging, given the number of options. This paper quantitatively evaluates the aforementioned technologies, outlining their main advantages and disadvantages and enabling the appropriate choice of the technology for an IWSAN of interest, considering the specific latency, reliability, scalability, energy consumption, data rate and coverage requirements.

Author Contributions: Conceptualization, A.S., E.D.P. and J.H.; validation, A.S., J.F., E.D.P. and J.H.; investigation, A.S.; resources, A.S., J.F., E.D.P. and J.H.; writing-original draft preparation, A.S.; writing-review and editing, A.S., J.F., E.D.P. and J.H.; visualization, A.S., J.F.; supervision, E.D.P. and J.H.; project administration, E.D.P. and J.H.; funding acquisition, E.D.P. and J.H. All authors have read and agreed to the published version of the manuscript.

Funding: This research was funded by Fund for Scientific Research-Flanders (FWO-V) grant number S004017N in the context of Strategic Basic Research (SBO) project IDEAL-IoT (Intelligent DEnse And Longe range IoT networks) project.

Acknowledgments: The authors would like to thank Serena Santi, Ashish Kumar Sultania, Glenn Daneels and Jetmir Haxhibeqiri for assisting in setting up the energy consumption measurement experiments.

Conflicts of Interest: The authors declare no conflict of interest.

\section{References}

1. Willig, A.; Matheus, K.; Wolisz, A. Wireless technology in industrial networks. Proc. IEEE 2005, 93, 1130-1151. [CrossRef]

2. Luvisotto, M.; Pang, Z.; Dzung, D. High-performance wireless networks for industrial control applications: New targets and feasibility. Proc. IEEE 2019, 107, 1074-1093. [CrossRef]

3. Weiner, M.; Jorgovanovic, M.; Sahai, A.; Nikolié, B. Design of a low-latency, high-reliability wireless communication system for control applications. In Proceedings of the IEEE International Conference on Communications (ICC), Sydney, Australia, 10-14 June 2014. [CrossRef]

4. Wei, Y.H.; Leng, Q.; Han, S.; Mok, A.K.; Zhang, W.; Tomizuka, M.; Li, T.; Malone, D.; Leith, D. RT-WiFi: Real-time high speed communication protocol for wireless control systems. ACM SIGBED Rev. 2013, 10, 28. [CrossRef]

5. Das Neves Valadão, Y.; Künzel, G.; Müller, I.; Pereira, C.E. Industrial Wireless Automation: Overview and Evolution of WIA-PA. IFAC-PapersOnLine 2018, 51, 175-180. [CrossRef]

6. HART Communications Foundation. HART Field Communication Protocol Specification; HFC_SPEC-12, Revision 6.0; HART Communications Foundation: Austin, TX, USA, 2001.

7. International Society of Automation (ISA) Standard. Wireless Systems for Industrial Automation: Process Control and Related Applications; ISA-100.11 a-2009; International Society of Automation (ISA) Standard: Research Triangle Park, NC, USA, 2009.

8. Harada, H.; Mizutani, K.; Fujiwara, J.; Mochizuki, K.; Obata, K.; Okumura, R. IEEE 802.15. 4g based Wi-SUN communication systems. IEICE Trans. Commun. 2017, 100, 1032-1043. [CrossRef]

9. Hermeto, R.T.; Gallais, A.; Theoleyre, F. Scheduling for IEEE802. 15.4-TSCH and slow channel hopping MAC in low power industrial wireless networks: A survey. Comput. Commun. 2017, 114, 84-105. [CrossRef]

10. IEC-International Electrotechnical Commission. Industrial Networks: Wireless Communication Network and Communication Profiles; IEC PAS 62601; IEC-International Electrotechnical Commission: Geneva, Switzerland, 2015.

11. Saelens, M.; Hoebeke, J.; Shahid, A.; De Poorter, E. Impact of EU duty cycle and transmission power limitations for sub-GHz LPWAN SRDs: An overview and future challenges. EURASIP J. Wirel. Commun. Netw. 2019, 2019, 219. [CrossRef]

12. Åkerberg, J.; Gidlund, M.; Björkman, M. Future research challenges in wireless sensor and actuator networks targeting industrial automation. In Proceedings of the 2011 9th IEEE International Conference on Industrial Informatics (INDIN), Lisbon, Portugal, 26-29 July 2011; pp. 410-415.

13. Ovsthus, K.; Kristensen, L.M. An industrial perspective on wireless sensor networks-a survey of requirements, protocols, and challenges. IEEE Commun. Surv. Tutor. 2014, 16, 1391-1412. 
14. Frotzscher, A.; Wetzker, U.; Bauer, M.; Rentschler, M.; Beyer, M.; Elspass, S.; Klessig, H. Requirements and current solutions of wireless communication in industrial automation. In Proceedings of the 2014 IEEE International Conference on Communications Workshops (ICC), Sydney, Australia, 10-14 June 2014; pp. 67-72.

15. Li, X.; Li, D.; Wan, J.; Vasilakos, A.V.; Lai, C.F.; Wang, S. A review of industrial wireless networks in the context of industry 4.0. Wirel. Netw. 2017, 23, 23-41. [CrossRef]

16. Wollschlaeger, M.; Sauter, T.; Jasperneite, J. The future of industrial communication: Automation networks in the era of the internet of things and industry 4.0. IEEE Ind. Electron. Mag. 2017, 11, 17-27. [CrossRef]

17. Park, P.; Ergen, S.C.; Fischione, C.; Lu, C.; Johansson, K.H. Wireless network design for control systems: A survey. IEEE Commun. Surv. Tutor. 2018, 20, 978-1013. [CrossRef]

18. Gunatilaka, D. High Performance Wireless Sensor-Actuator Networks for Industrial Internet of Things. Ph.D. Thesis, Washington University in St. Louis, St. Louis, MO, USA, 2019.

19. Wang, Q.; Jiang, J. Comparative examination on architecture and protocol of industrial wireless sensor network standards. IEEE Commun. Surv. Tutor. 2016, 18, 2197-2219. [CrossRef]

20. De Pellegrini, F.; Miorandi, D.; Vitturi, S.; Zanella, A. On the use of wireless networks at low level of factory automation systems. IEEE Trans. Ind. Inf. 2006, 2, 129-143. [CrossRef]

21. Christin, D.; Mogre, P.S.; Hollick, M. Survey on wireless sensor network technologies for industrial automation: The security and quality of service perspectives. Future Internet 2010, 2, 96-125. [CrossRef]

22. Lu, C.; Saifullah, A.; Li, B.; Sha, M.; Gonzalez, H.; Gunatilaka, D.; Wu, C.; Nie, L.; Chen, Y. Real-time wireless sensor-actuator networks for industrial cyber-physical systems. Proc. IEEE 2016, 104, 1013-1024. [CrossRef]

23. Sanchez-Iborra, R.; Cano, M.D. State of the art in LP-WAN solutions for industrial IoT services. Sensors 2016, 16, 708. [CrossRef]

24. Varghese, A.; Tandur, D. Wireless requirements and challenges in Industry 4.0. In Proceedings of the 2014 International Conference on Contemporary Computing and Informatics (IC3I), Mysore, India, 27-29 November 2014; pp. 634-638.

25. Zurawski, R. Industrial Communication Technology Handbook; CRC Press: Boca Raton, FL, USA, 2014.

26. Tindell, K.; Burns, A. Guaranteeing message latencies on control area network (CAN). In Proceedings of the 1st International CAN Conference, 1994. Available online: https:/ / citeseer.ist.psu.edu/viewdoc/download? doi=10.1.1.43.3251\&rep=rep1\&type=pdf (accessed on 15 January 2020).

27. Luvisotto, M.; Tramarin, F.; Vitturi, S. Assessing the impact of full-duplex wireless in real-time industrial networks. In Proceedings of the IECON 2018 - 44th Annual Conference of the IEEE Industrial Electronics Society, Washington, DC, USA, 21-23 October 2018; pp. 4119-4124.

28. Vuran, M.C.; Akyildiz, I.F. Error control in wireless sensor networks: A cross layer analysis. IEEE/ACM Trans. Netw. (TON) 2009, 17, 1186-1199. [CrossRef]

29. Bor, M.C.; Roedig, U.; Voigt, T.; Alonso, J.M. Do LoRa low-power wide-area networks scale? In Proceedings of the 19th ACM International Conference on Modeling, Analysis and Simulation of Wireless and Mobile Systems, Malta, 13-17 November 2016; pp. 59-67.

30. Aref, M.; Sikora, A. Free space range measurements with Semtech LoRa ${ }^{\mathrm{TM}}$ technology. In Proceedings of the 2014 2nd International Symposium on Wireless Systems within the Conferences on Intelligent Data Acquisition and Advanced Computing Systems, Offenburg, Germany, 11-12 September 2014; pp. 19-23.

31. Van den Abeele, F.; Haxhibeqiri, J.; Moerman, I.; Hoebeke, J. Scalability analysis of large-scale LoRaWAN networks in ns-3. IEEE Internet Things J. 2017, 4, 2186-2198. [CrossRef]

32. Haxhibeqiri, J.; De Poorter, E.; Moerman, I.; Hoebeke, J. A survey of lorawan for iot: From technology to application. Sensors 2018, 18, 3995. [CrossRef]

33. Rizzi, M.; Ferrari, P.; Flammini, A.; Sisinni, E.; Gidlund, M. Using LoRa for industrial wireless networks. In Proceedings of the 2017 IEEE 13th International Workshop on Factory Communication Systems (WFCS), Trondheim, Norway, 31 May-2 June 2017; pp. 1-4.

34. Pötsch, A.; Hammer, F. Towards end-to-end latency of LoRaWAN: Experimental analysis and IIoT applicability. In Proceedings of the 2019 15th IEEE international workshop on factory communication systems (WFCS), Sundsvall, Sweden, 27-29 May 2019; pp. 1-4.

35. Šljivo, A.; Kerkhove, D.; Tian, L.; Famaey, J.; Munteanu, A.; Moerman, I.; Hoebeke, J.; De Poorter, E. Performance Evaluation of IEEE 802.11ah Networks With High-Throughput Bidirectional Traffic. Sensors 2018, 18, 325. [CrossRef] 
36. Seferagić, A.; Moerman, I.; De Poorter, E.; Hoebeke, J. Evaluating the suitability of IEEE 802.11 ah for low-latency time-critical control loops. IEEE Internet Things J. 2019, 6, 7839-7848. [CrossRef]

37. Santi, S.; Šljivo, A.; Tian, L.; De Poorter, E.; Hoebeke, J.; Famaey, J. Supporting heterogeneous IoT traffic using the IEEE 802.11 Ah restricted access window. In Proceedings of the 15th ACM Conference on Embedded Network Sensor Systems, Delft, The Netherlands, 6-8 November 2017; p. 32.

38. Šljivo, A.; Kerkhove, D.; Moerman, I.; De Poorter, E.; Hoebeke, J. Reliability and Scalability Evaluation with TCP/IP of IEEE802. 11ah Networks. In Proceedings of the Workshop on ns3 (WNS3), Porto, Portugal, 13-14 June 2017; pp. 1-4.

39. Chen, J.; Hu, K.; Wang, Q.; Sun, Y.; Shi, Z.; He, S. Narrowband internet of things: Implementations and applications. IEEE Internet Things J. 2017, 4, 2309-2314. [CrossRef]

40. Wang, Y.P.E.; Lin, X.; Adhikary, A.; Grovlen, A.; Sui, Y.; Blankenship, Y.; Bergman, J.; Razaghi, H.S. A primer on 3GPP narrowband Internet of Things. IEEE Commun. Mag. 2017, 55, 117-123. [CrossRef]

41. Basu, S.S.; Sultania, A.K.; Famaey, J.; Hoebeke, J. Experimental Performance Evaluation of NB-IoT. In Proceedings of the International Conference on Wireless and Mobile Computing, Networking and Communications (WiMob), Barcelona, Spain, 21-23 October 2019.

42. Martinez, B.; Adelantado, F.; Bartoli, A.; Vilajosana, X. Exploring the Performance Boundaries of NB-IoT. IEEE Internet Things J. 2019, 6, 5702-5712 [CrossRef]

43. Martinez, B.; Vilajosana, X.; Chraim, F.; Vilajosana, I.; Pister, K.S. When scavengers meet industrial wireless. IEEE Trans. Ind. Electron. 2015, 62, 2994-3003. [CrossRef]

44. Petersen, S.; Carlsen, S. Performance evaluation of WirelessHART for factory automation. In Proceedings of the 2009 IEEE Conference on Emerging Technologies \& Factory Automation, Palma de Mallorca, Spain, 22-25 September 2009; pp. 1-9.

45. Han, S.; Zhu, X.; Aloysius, K.M.; Nixon, M.; Blevins, T.; Chen, D. Control over WirelessHART network. In Proceedings of the IECON 2010-36th Annual Conference on IEEE Industrial Electronics Society, Glendale, AZ, USA, 7-10 November 2010; pp. 2114-2119.

46. Åkerberg, J.; Gidlund, M.; Reichenbach, F.; Björkman, M. Measurements on an Industrial Wireless Hart Network Supporting Profisafe: A Case Study. In Proceedings of the International Conference on Emerging Technologies and Factory Automation (ETFA), Toulouse, France, 5-9 September 2011; IEEE, pp. 1-8.

47. Hassan, S.M.; Bingi, K.; Ibrahim, R.; Chein, L.J.; Supramaniam, T. Implementation of flow control over wirelessHART sensor network using wirelessHART adaptors. Indones. J. Electr. Eng. Comput. Sci. 2019, 15, 910-919.

48. One Wireless Field Device Access Point Specification Release 310 OW03-650-310 Kernel Description. 2018. Available online: https:/ / www.honeywellprocess.com/library/marketing/tech-specs/onewireless-fdapspecification.pdf (accessed on 14 December 2018).

49. Jecan, E.; Pop, C.; Padrah, Z.; Ratiu, O.; Puschita, E. A dual-standard solution for industrial Wireless Sensor Network deployment: Experimental testbed and performance evaluation. In Proceedings of the 14th IEEE International Workshop on Factory Communication Systems (WFCS), Imperia, Italy, 13-15 June 2018.

50. Karvonen, H.; Pomalaza-Ráez, C.; Mikhaylov, K.; Hämäläinen, M.; Iinatti, J. Experimental Performance Evaluation of BLE 4 Versus BLE 5 in Indoors and Outdoors Scenarios; Advances in Body Area Networks I; Fortino, G.; Wang, Z., Eds.; Springer: Cham, Switzerland, 2019; pp. 235-251.

51. Baert, M.; Rossey, J.; Shahid, A.; Hoebeke, J. The Bluetooth mesh standard: An overview and experimental evaluation. Sensors 2018, 18, 2409. [CrossRef]

52. Mochizuki, K.; Obata, K.; Mizutani, K.; Harada, H. Development and field experiment of wide area Wi-SUN system based on IEEE 802.15. 4g. In Proceedings of the 2016 IEEE 3rd World Forum on Internet of Things (WF-IoT), Reston, VA, USA, 12-14 December 2016; pp. 76-81.

53. Cena, G.; Scanzio, S.; Valenzano, A.; Zunino, C. Experimental Analysis and Comparison of Industrial IoT Devices based on TSCH. In Proceedings of the 24th IEEE International Conference on Emerging Technologies and Factory Automation (ETFA), Zaragoza, Spain, 10-13 September 2019. [CrossRef]

54. ETSI. System Reference document (SRdoc). In Technical characteristics for Low Power Wide Area Networks Chirp Spread Spectrum (LPWAN-CSS) operating in the UHF spectrum below 1 GHz; ETSI TR 103526 V1.1.1; ETSI: Sophia Antipolis Cedex, France, 2018. 
55. LoRa Alliance. LoRaWAN ${ }^{\mathrm{TM}}$ Specification; LoRa Alliance Inc., Beaverton, OR, USA, 2015. Available online: https:/ /lora-alliance.org/sites/default/files/2018-04/lorawantm_specification_-v1.1.pdf (accessed on 11 October 2017).

56. IEEE Standard for Information Technology-Telecommunications and Information Exchange between Systems-Local and Metropolitan Area Networks-Specific Requirements-Part 11: Wireless LAN Medium Access Control (MAC) and Physical Layer (PHY) Specifications Amendment 2: Sub 1 GHz License Exempt Operation; IEEE Std 802.11ah-2016 (Amendment to IEEE Std 802.11-2016, as amended by IEEE Std 802.11ai-2016); IEEE: New York, NY, USA 2017; pp. 1-594. [CrossRef]

57. Liu, Y.; Guo, J.; Orlik, P.; Nagai, Y.; Watanabe, K.; Sumi, T. Coexistence of 802.11 ah and 802.15. 4g networks. In Proceedings of the 2018 IEEE Wireless Communications and Networking Conference (WCNC), Barcelona, Spain, 15-18 April 2018; pp. 1-6.

58. Haxhibeqiri, J.; Van den Abeele, F.; Moerman, I.; Hoebeke, J. LoRa scalability: A simulation model based on interference measurements. Sensors 2017, 17, 1193. [CrossRef]

59. Sultania, A.K.; Delgado, C.; Famaey, J. Implementation of NB-IoT Power Saving Schemes in ns-3. In Proceedings of the 2019 Workshop on Next-Generation Wireless with ns-3, Florence, Italy, 21 June 2019; pp. 5-8.

60. Tian, L.; Šljivo, A.; Santi, S.; De Poorter, E.; Hoebeke, J.; Famaey, J. Extension of the IEEE 802.11 ah ns-3 Simulation Module. In Proceedings of the 10th Workshop on ns-3, Mangalore, India, 13-14 June 2018; pp. 53-60.

61. Municio, E.; Daneels, G.; Vučinić, M.; Latré, S.; Famaey, J.; Tanaka, Y.; Brun, K.; Muraoka, K.; Vilajosana, X.; Watteyne, T. Simulating 6TiSCH networks. Trans. Emerg. Telecommun. Technol. 2019, 30, e3494. [CrossRef]

62. Muñoz, J.; Chang, T.; Vilajosana, X.; Watteyne, T. Evaluation of IEEE802. $15.4 \mathrm{~g}$ for Environmental Observations. Sensors 2018, 18, 3468. [CrossRef]

63. Bellekens, B.; Tian, L.; Boer, P.; Weyn, M.; Famaey, J. Outdoor IEEE 802.11 ah range characterization using validated propagation models. In Proceedings of the GLOBECOM 2017-2017 IEEE Global Communications Conference, Singapore, 4-8 December 2017; pp. 1-6.

64. Ousat, B.; Ghaderi, M. LoRa Network Planning: Gateway Placement and Device Configuration. In Proceedings of 2019 IEEE International Congress on Internet of Things (ICIOT), Milan, Italy, 8-13 July 2019; pp. 25-32.

65. Tian, L.; Khorov, E.; Latré, S.; Famaey, J. Real-Time Station Grouping under Dynamic Traffic for IEEE 802.11ah. Sensors 2017, 17, 1559. [CrossRef] [PubMed]

66. Khorov, E.; Lyakhov, A.; Krotov, A.; Guschin, A. A survey on IEEE 802.11ah: An enabling networking technology for smart cities. Comput. Commun. 2015, 58, 53-69. [CrossRef]

67. Shamsi, M. WirelessHART Field Device Installation and Configuration. 2016. Available online: https:/ / www.emerson.com/documents/automation/article-wirelesshart-field-device-installationconfiguration-en-38282.pdf (accessed on 8 January 2019).

(C) 2020 by the authors. Licensee MDPI, Basel, Switzerland. This article is an open access article distributed under the terms and conditions of the Creative Commons Attribution (CC BY) license (http://creativecommons.org/licenses/by/4.0/). 